\title{
Radio-Continuum study of the Nearby Sculptor Group Galaxies. Part 1: NGC 300 at $\lambda=20 \mathrm{~cm}$
}

\author{
Timothy J. Galvin, Miroslav D. Filipović, Evan J. \\ Crawford, Graeme Wong, Jeff L. Payne, Ain De \\ Horta, Graeme L. White ${ }^{1}$, Nick Tothill, Danica \\ Drašković • \\ Thomas G. Pannuti, Caleb K. Grimes, Benjamin J. Cahall \\ - William C. Millar² • Seppo Laine
}

\begin{abstract}
A series of new radio-continuum $(\lambda=20 \mathrm{~cm})$ mosaic images focused on the NGC 300 galactic system were produced using archived observational data from the VLA and/or ATCA. These new images are both very sensitive $(\mathrm{rms}=60 \mu \mathrm{Jy})$ and feature high angular resolution $\left(<10^{\prime \prime}\right)$. The most prominent new feature is the galaxy's extended radio-continuum emission, which does not match its optical appearance. Using these newly created images a number of previously unidentified discrete sources have been discovered. Furthermore, we demonstrate that a joint deconvolution approach to imaging this complete data-set is inferior when compared to an IMMERGE approach.
\end{abstract}

\section{Introduction}

At 1.9 Mpc away (Rizzi et al. 2006), NGC 300 is the closest spiral galaxy of the nearby Sculptor Group.

Timothy J. Galvin, Miroslav D. Filipović, Evan J. Crawford, Graeme Wong, Jeff L. Payne, Ain De Horta, Graeme L. White, Nick Tothill, Danica Drašković

University of Western Sydney, Locked Bag 1797, Penrith South DC, NSW 1797, Australia

Thomas G. Pannuti, Caleb K. Grimes, Benjamin J. Cahall

Department of Earth and Space Sciences, Space Science Center, 235 Martindale Drive, Morehead State University, Morehead, KY 40351, USA

William C. Millar

Centre for Astronomy, James Cook University, Townsville, Queensland 4811, Australia

Seppo Laine

Spitzer Science Center, California Institute of Technology, MS 220-6, Pasadena, CA 91125, USA

${ }^{1}$ Charles Sturt University, School of Dentistry, Wagga Wagga NSW, Australia

${ }^{2}$ Grand Rapids Community College, 143 Bostwick N.E., Grand Rapids, MI, 49503, USA
This proximity is an advantage because it allows for this galaxy to be examined in great detail. Previous radio-continuum and optical studies of NGC 300 (Pannuti et al. 2000: Payne et al. 2004: Millar et al. 2011) utilised either the Australia Telescope Compact Array (ATCA) or the Very Large Array (VLA) as their primary instrument. However, these past studies suffer from either low resolution, poor sensitivity or both.

Until the next generation radio telescopes, such as the Australian Square Kilometre Array Pathfinder (ASKAP), Karoo Array Telescope (KAT \& MeerKAT) and Square Kilometre Array (SKA), become operational we are restricted to consolidating a selection of NGC 300 radio observations. In this paper, we reexamine all available archived radio-continuum observations performed at ATCA and VLA at $\lambda=20 \mathrm{~cm}(\nu=1.4 \mathrm{GHz})$ with the intention of merging these observations into a single radio-continuum image. By combining a large amount of existing data using the latest generation of computer power we can create new images that feature both high angular resolution and excellent sensitivity. The newly constructed images are analysed and the difference between the various NGC 300 images created at $20 \mathrm{~cm}$ are discussed.

In $\S 2$ we describe the observational data and reduction techniques. In $\S 3$ we present our new maps, a brief discussion is given in $\S 4$, and $\S 5$ is the conclusion.

\section{OBSERVATIONAL DATA}

\subsection{Observational Data}

To create a high-resolution and sensitive radio-continuum image a number (5) of observations from the ATCA and VLA were used. These observations were selected from the Australian Telescope Online Archive (ATOA) and the National Radio Astronomy Observatory (NRAO) 
Science Data Archive. The observations which were selected and used here are summarised in Table 1. Because NGC 300 is far south, the VLA could only record data for short durations (see Table 1) and therefore over limited hour angle ranges.

ATCA project C1757 was conducted in mosaic mode with a total of eight pointings being observed over eight days. This project mapped the neutral hydrogen emission of NGC 300 (Westmeier et al.|2011), which extends significantly beyond the galaxy's optical emission. We used only the four innermost pointings which were directly centred on NGC 300. Other VLA and ATCA observations used in this study consist of single pointings of NGC 300. All images are primary beam corrected.

For both ATCA projects, the source PKS 1934-638 was used as the primary calibrator. The sources PKS 0008-421 and PKS 0823-500 were used as secondary calibrators for ATCA projects $\mathrm{C} 1757$ and C828 respectively. VLA observations AC101 and AL445 used source $0134+329$ (IAU J0137+3309) as their primary calibrator, while sources 0023-263 (IAU J0025-2602) and 0022-423 (IAU J0024-4202) were used as the secondary calibrator, respectively. Project AC308 used 0521+166 (PKS J0521+1638) as its primary calibrator and source 0116-208 (PKS J0116-2052) as its secondary calibrator.

\subsection{Image Creation}

To create the best possible NGC 300 mosaic image at $20 \mathrm{~cm}$, we examined the radial distance in the $u v$-plane of all observations combined (Fig. 1) and found that the $u v$-plane is densely sampled up to $\sim 30 \mathrm{k} \lambda$, but quite sparsely sampled at longer baselines. Thus we restricted our imaging to the $0-30 \mathrm{k} \lambda$ range and discarded longer baselines in VLA observations.

The MIRIAD (Sault and Killeen 2006), AIPS (Greisen 2010) and KARMA (Gooch 2006) software packages were used for data reduction and analysis. Because of the large volume of data, the MIRIAD package was compiled to run on a 16-processor high-performance computer system.

Initially, observations which were performed using the VLA were imported into AIPS using the task FILLM, and then all sources were split with SPLIT. Using the task UVFIX, source coordinates were converted from the B1950 to the J2000 reference frame and the task FITTP was used to export each source to a FITs file.

The MIRIAD package was then used for actual data reduction. The task ATLOD was used to convert ATCA observations into MIRIAD files, while the task FITS was used to import the previous AIPS-produced fits files and convert them to MIRIAD files.
Typical calibration and flagging procedures were then carried out (Sault and Killeen 2006). Using the task INVERT, two mosaic images (one based on ATCA data and the other on VLA data) were created using a natural weighting scheme. Each mosaic image was then cleaned using the task MOSSDI. The MOSSDI task is a SDI clean algorithm designed for mosaic images (Steer et al. 1984). To convolve a clean model the task RESTOR was then used on each of the cleaned maps.

The restored mosaic images were then merged together using the task IMMERGE. This task uses a linear approach to merge images of a different resolution, where the low resolution image is assumed to better represent short spacing, and the higher resolution image to better represent the fine structure. IMMERGE changes the weighting by the normalisation process in the overlapping region.

We also created a single mosaic image which was comprised of all selected ATCA and VLA projects using the task INVERT. The dirty map was then cleaned with MOSSDI before being convolved with RESTOR.

The difference between the two approaches to data combination is that the single image (in which all $u v$ data are inverted simultaneously) contains all calibration uncertainties and weightings from each data set, while the image produced by merging individual images normalises the different calibrations in regions of uv overlap.

The ATCA bandpass comprises 13 channels, one of which is contaminated by $\mathrm{HI}$ emission. From the H I results of Westmeier et al. (2011), the H I contamination can be seen to be restricted to channel 4, which was flagged out and does not contribute to our images.

\section{RESULTS}

When comparing individual maps created from each observation, the different effects of varying array configurations can be seen. The mosaic image produced from C1757 (Fig. 22) showed a much better defined region of extended emission. However, it lacked the high resolution of project C828 (Fig. 3), due to the shorter baselines of the $\mathrm{C} 1757$ project.

VLA observation AL445 (Fig. 4) was performed using the longest baseline, giving this image the highest resolution among all the individual images presented in this paper. As expected, point sources dominate this field. The other VLA observations, AC101 (Fig. 5) and AC308 (Fig. 6), were taken with much smaller array configurations, resulting in lower-resolution images with significant extended emission. Both of these observations have short integration times. AC308 had only 36 seconds integration, leading to a very shallow image. 
Table 1 List of VLA and ATCA observations of NGC 300 used in this study. RA and DEC represent coordinates of central pointings.

\begin{tabular}{|c|c|c|c|c|c|c|c|c|c|c|}
\hline $\begin{array}{l}\text { Project } \\
\text { Code }\end{array}$ & $\begin{array}{l}R A(J 2000) \\
\mathrm{h} \text { m s }\end{array}$ & $\begin{array}{c}\operatorname{Dec}(J 2000) \\
\circ,,\end{array}$ & Dates & Instrument & $\begin{array}{l}\text { Array } \\
\text { Type }\end{array}$ & $\begin{array}{c}\nu \\
(M H z)\end{array}$ & $\begin{array}{c}\Delta \nu \\
(M H z)\end{array}$ & $\begin{array}{c}\text { Duration } \\
\text { (hours) }\end{array}$ & $\begin{array}{c}\text { Primary } \\
\text { Calibrator }\end{array}$ & $\begin{array}{l}\text { Secondary } \\
\text { Calibrator }\end{array}$ \\
\hline AL445 & 005453.20 & $-3740 \quad 57.00$ & $13 / 06 / 1998$ & VLA & $\mathrm{AB}$ & 1435,1465 & 15,15 & 1 & $0134+329$ & $0022-423$ \\
\hline $\mathrm{AC} 101$ & 005452.72 & $\begin{array}{lll}-37 & 41 & 09.02\end{array}$ & $13 / 07 / 1984$ & VLA & $\mathrm{CD}$ & 1465,1515 & 50,50 & 0.1 & $0134+329$ & 0023-263 \\
\hline AC308 & 005530.00 & -375154.00 & 09/10/1993 & VLA & $\mathrm{CD}$ & 1365,1435 & 50,50 & 0.01 & $0521+166$ & 0116-208 \\
\hline $\mathrm{C} 828$ & 005453.47 & -374100.00 & $27-28 / 02 / 2000$ & ATCA & $6 \mathrm{~A}$ & 1384 & 128 & 10.50 & $1934-638$ & $0823-500$ \\
\hline C1757 & 005411.81 & -373249.00 & $20 / 11 / 2007$ & ATCA & EW367 & 1384 & 128 & 1.04 & $1934-638$ & $0008-421$ \\
\hline C1757 & 005411.81 & -373249.00 & $02 / 02 / 2008$ & ATCA & EW367 & 1384 & 128 & 1.15 & $1934-638$ & $0008-421$ \\
\hline $\mathrm{C} 1757$ & $00 \quad 5535.19$ & $\begin{array}{lll}-37 & 49 & 19.00\end{array}$ & $03 / 02 / 2008$ & ATCA & EW367 & 1384 & 128 & 1.14 & $1934-638$ & $0008-421$ \\
\hline C1757 & 005535.19 & $\begin{array}{lll}-37 & 32 & 49.00\end{array}$ & $23 / 05 / 2008$ & ATCA & EW 367 & 1384 & 128 & 1.22 & $1934-638$ & $0008-421$ \\
\hline C1757 & 005411.81 & $\begin{array}{lll}-37 & 49 & 19.00\end{array}$ & $24 / 05 / 2008$ & ATCA & EW367 & 1384 & 128 & 1.33 & $1934-638$ & $0008-421$ \\
\hline C1757 & $00 \quad 5535.19$ & $\begin{array}{lll}-37 & 49 & 19.00\end{array}$ & $13 / 11 / 2008$ & ATCA & EW367 & 1384 & 128 & 1.17 & $1934-638$ & $0008-421$ \\
\hline C1757 & 005535.19 & $\begin{array}{lll}-37 & 32 & 49.00\end{array}$ & $17 / 11 / 2008$ & ATCA & EW 367 & 1384 & 128 & 1.28 & $1934-638$ & $0008-421$ \\
\hline $\mathrm{C} 1757$ & $00 \quad 54 \quad 11.81$ & -374919.00 & $19 / 11 / 2008$ & ATCA & EW 367 & 1384 & 128 & 1.23 & $1934-638$ & $0008-421$ \\
\hline
\end{tabular}

\section{I $1.4320 \mathrm{GHz}$}

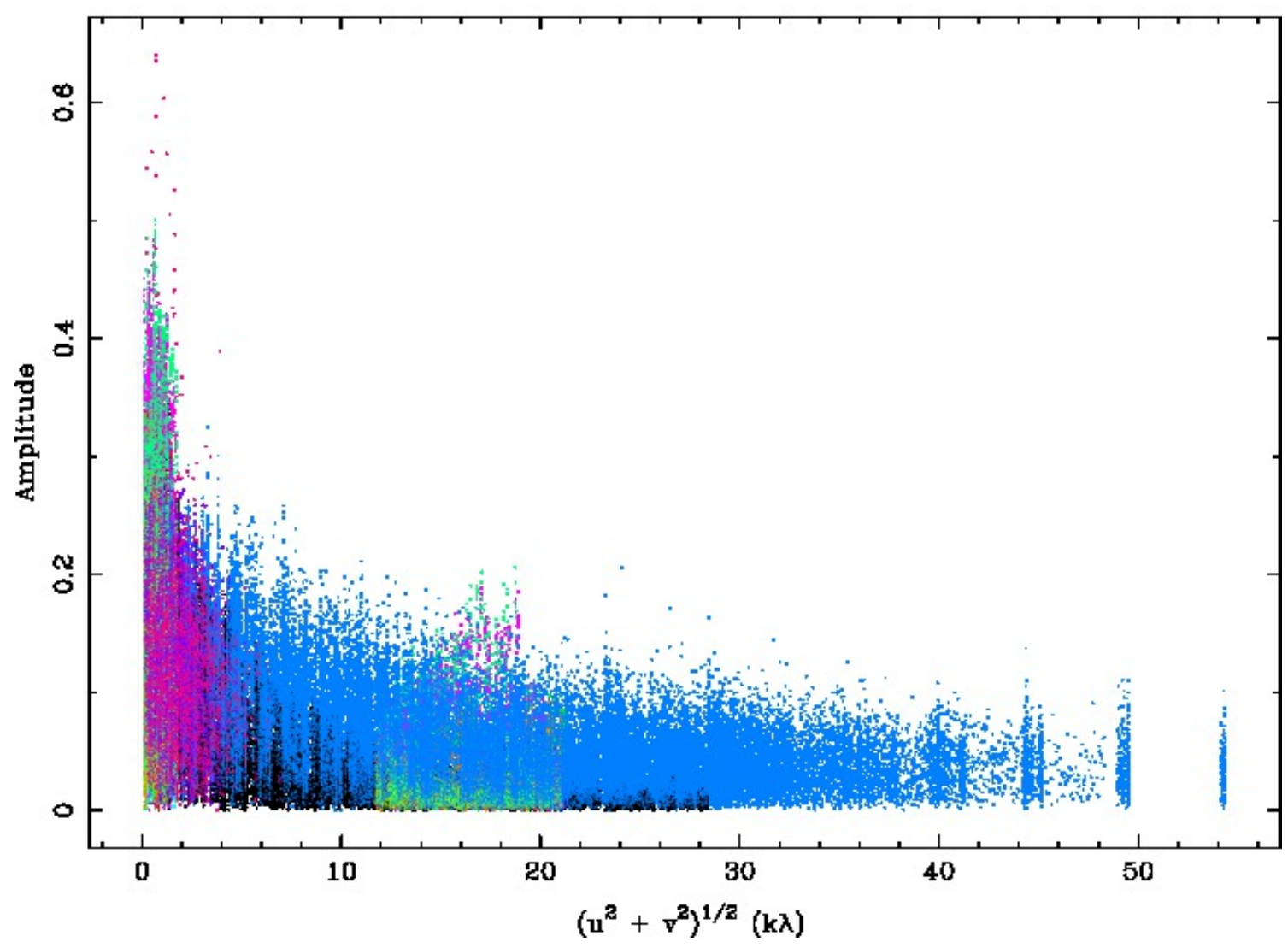

Fig. 1 Graph showing the unrestricted amplitude vs $u v$-distance of all ATCA/VLA projects used in this work. Each project is represented as different colour in this graph. Here, project C828 is dark blue, project C1757 is green, project AL445 is light blue, project AC308 is pink and project AC101 is purple. 
Fig. 7 is a mosaic image containing both ATCA observations C1757 and C828. It shows a well-defined region of extended emission with a number of definitive point sources. We also created a mosaic image comprised of VLA observations AL445, AC308 and AC101 (Fig. 8). Although there is no obvious extended emission in this image, there are a number of resolved point sources.

In Fig. 9 we show the mosaic image produced with all five observations. Although there is a clear region of extended emission and a number of resolved point sources, this image suffers from significant side-lobe distortion. Finally, in Fig. 10 we present the result of merging together each mosaic image. We note a well-defined region of extended emission in this image, with a total integrated flux density estimated to be $4.62 \pm 0.01 \mathrm{Jy}$ at $20 \mathrm{~cm}$. The details of all new images presented here, including the various combined images, are summarised in Table2, Images were also produced using a uniform weighing scheme, but were disregarded because of the absence of the extended emission structure.

Table 2 The details of ATCA/VLA single and merged projects of NGC 300 mosaics at $20-\mathrm{cm}$.

\begin{tabular}{lccc}
\hline $\begin{array}{l}\text { ATCA/VLA } \\
\text { Project }\end{array}$ & $\begin{array}{c}\text { Beam Size } \\
(\operatorname{arcsec})\end{array}$ & $\begin{array}{c}\text { r.m.s. } \\
(\mathrm{mJy} / \text { beam })\end{array}$ & Figure \\
\hline C1757 & $153.1 \times 72.5$ & 0.30 & 2 \\
C828 & $13.8 \times 10.1$ & 0.07 & 3 \\
AL445 & $7.0 \times 4.5$ & 0.15 & 4 \\
AC101 & $77.9 \times 36.7$ & 0.30 & 5 \\
AC308 & $77.8 \times 44.7$ & 0.50 & 6 \\
All ATCA & $13.8 \times 10.1$ & 0.07 & 7 \\
All VLA & $10.9 \times 4.8$ & 0.09 & 8 \\
All (invert) & $11.8 \times 5.0$ & 0.13 & 9 \\
All (merged) & $10.8 \times 4.8$ & 0.06 & 10 \\
\hline
\end{tabular}

\section{DISCUSSION}

\subsection{Discrete Sources Within The Field Of NGC 300}

Our most sensitive and highest-resolution image is shown in Fig. 10. Using this image, a total of 72 radio sources above $3 \sigma(0.18 \mathrm{mJy} /$ beam $)$ were identified within the area defined by Payne et al. (2004) (Table3). All sources identified in this study were (Gaussian) fitted without subtracting the background which changes across the field. While the uncertainty is dependent on the flux density of a source (with larger uncertainties for weaker sources) we estimate that the overall uncertainty in source flux density is less than $10 \%$ (see $§ 4.2$ ).

A previous radio-continuum study of NGC 300 was able to identify a total of 47 sources at the wavelength of
$20 \mathrm{~cm}$ (Payne et al. 2004; combined Cols. 5 and 6 from Table 4). These sources were found by combining two independent observations by the ATCA and the VLA. Eleven of these 47 sources could not be identified in our final image. This difference can be attributed to the method of identifying sources which the previous study adopted. For a source to be considered real, it only had to appear in one of the two radio-continuum images. Of the eleven sources which were found not to be in our final image, none could be identified in both $20 \mathrm{~cm}$ images which were produced in the previous studies. This potentially indicates that these eleven sources are not real as they can only be found in a single $20 \mathrm{~cm}$ radiocontinuum image. Alternatively, they may represent some sort of transient or variable sources (e.g. QSOs) with very low flux densities of around $3 \sigma$. Indeed, we found some seven sources (Table 3 , difference between the new flux densities (Col. 4) and previous (Cols. 5 or $6)$ ) with very different flux densities $(>20 \%)$ indicating a small but significant population of variable sources.

We also compared our radio-continuum catalogue with a list of 28 well-established optically identified H II regions in NGC 300 (Bresolin et al. 2009). We note that 11 of our radio sources (sources \# 2, 11, 18, $21,25,28,34,39,51,52$ and 54) coincide (to within $\left.5^{\prime \prime}\right)$ with Hil regions from this list. Our source \# 39 (ATCA J005451.7-373940) was previously classified as a SNR in Payne et al. (2004).

In Fig. 11 we show the flux density distribution of all radio-continuum sources found in this study (Table 3 . Col. 3). As expected, the majority of sources (85\%) are within $10 \sigma$ flux density level.

\subsection{Comparison of Discrete Source Flux Densities}

While our flux densities are highly-correlated with those of Payne et al. (2004), they are systematically higher. Assuming no systematic offset between the two sets of flux densities, we find that our flux densities (Col. 4 of Table 3) are about $20 \%$ greater than those reported by Payne et al. (2004) (ATCA observations in Col. 5 and VLA in Col. 6). The ATCA and VLA flux densities of Payne et al. (2004) (Cols. 5 and 6) are in good internal agreement with ATCA/VLA $=1.07 \pm 0.06$ (standard error of mean for 33 sources in common). However, our new determinations of flux densities are higher than those of Payne et al. (2004) by $19 \pm 5 \%$ : For the 8 sources from our study (Col. 4) which are stronger than $1 \mathrm{mJy}$, four sources (\# 9, 24, 42 and 62) had corresponding flux densities listed by Payne et al. (2004) (Cols. 5 and 6). We found that from these four sources our flux densities are $23 \pm 9 \%$ higher than the average of the ATCA and VLA observations; for all 35 sources 


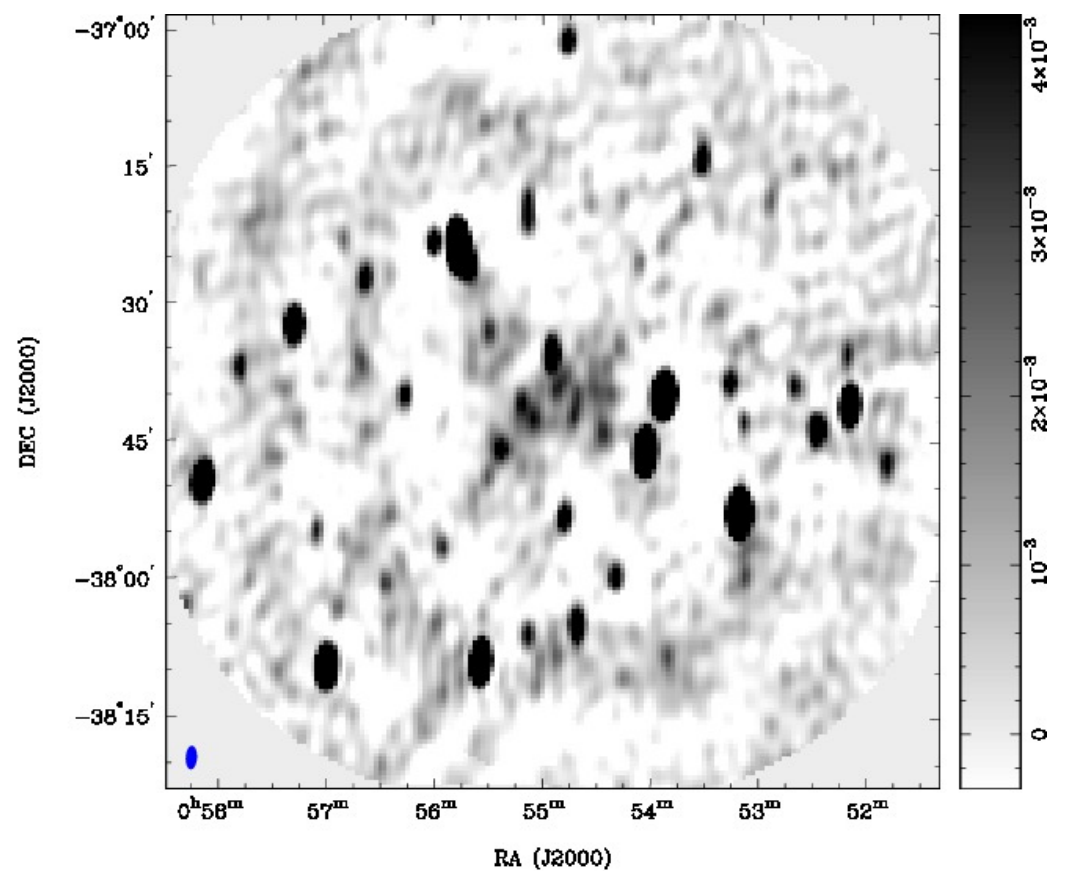

Fig. 2 ATCA Project C1757 mosaic radio-continuum total intensity image of NGC 300 . The synthesised beam, as represented by the blue ellipse in the lower left hand corner, is $153.1^{\prime \prime} \times 72.5^{\prime \prime}$ and the r.m.s. noise is $0.30 \mathrm{mJy} / \mathrm{beam}$. This image is in terms of Jy/Beam.

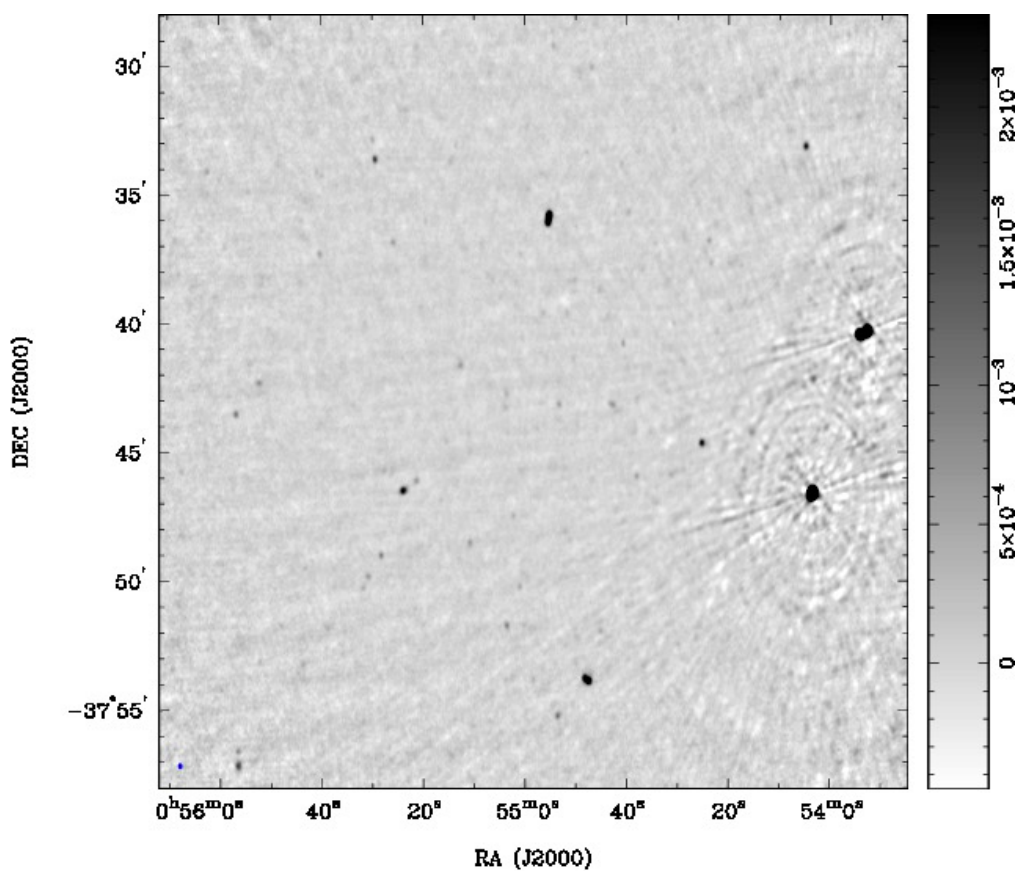

Fig. 3 ATCA Project C828 radio-continuum total intensity image of NGC 300. The synthesised beam, as represented by the blue ellipse in the lower left hand corner, is $13.8^{\prime \prime} \times 10.1^{\prime \prime}$ and the r.m.s noise is $0.07 \mathrm{mJy} /$ beam. This image is in terms of Jy/Beam. 


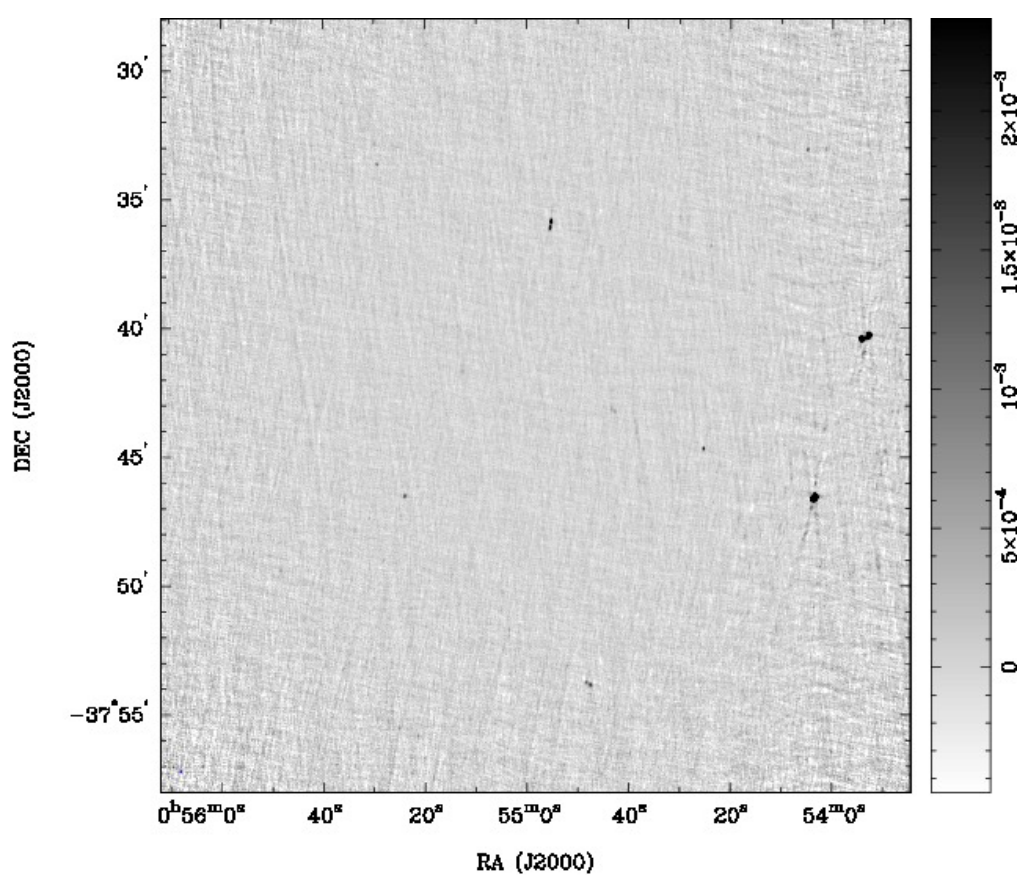

Fig. 4 VLA Project AL0445 radio-continuum total intensity image of NGC 300. The synthesised beam, as represented by the blue ellipse in the lower left hand corner, is $7.0^{\prime \prime} \times 4.5^{\prime \prime}$ and the r.m.s noise is $0.15 \mathrm{mJy} /$ beam. This image is in terms of Jy/Beam.

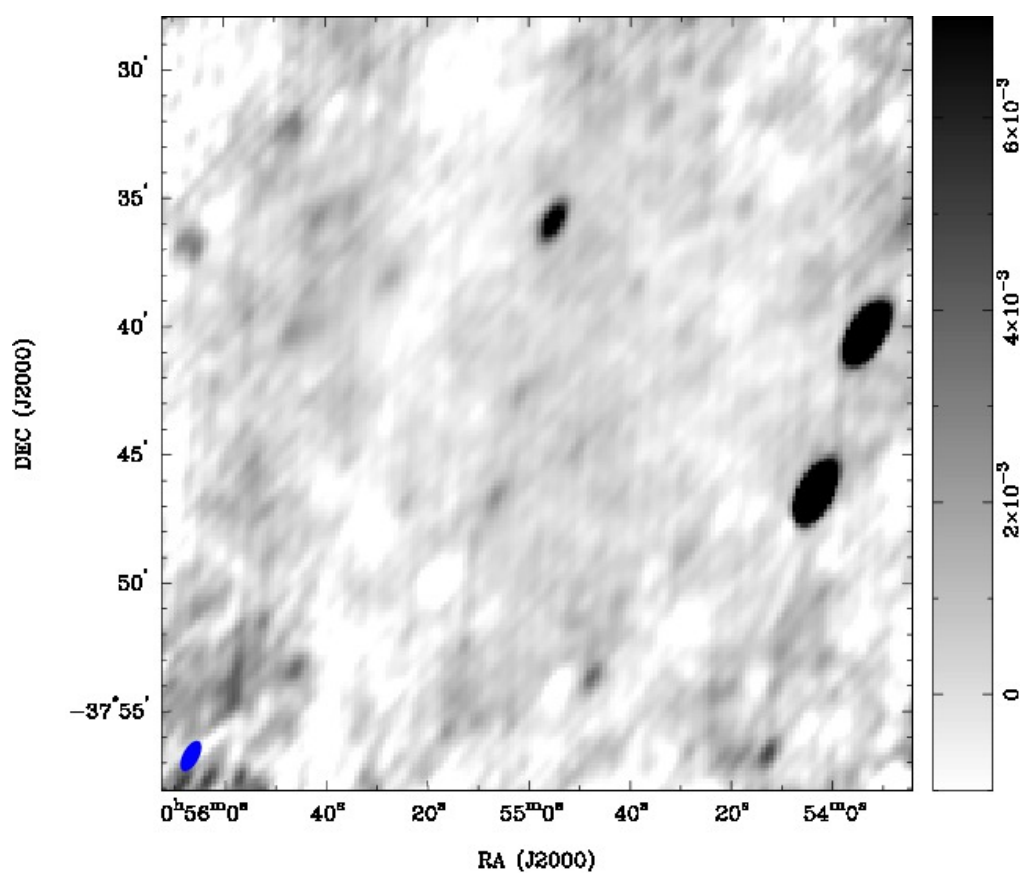

Fig. 5 VLA Project AC0101 radio-continuum total intensity image of NGC 300. The synthesised beam, as represented by the blue ellipse in the lower left hand corner, is $77.9^{\prime \prime} \times 36.7^{\prime \prime}$ and the r.m.s noise is $0.30 \mathrm{mJy} /$ beam. This image is in terms of Jy/Beam. 


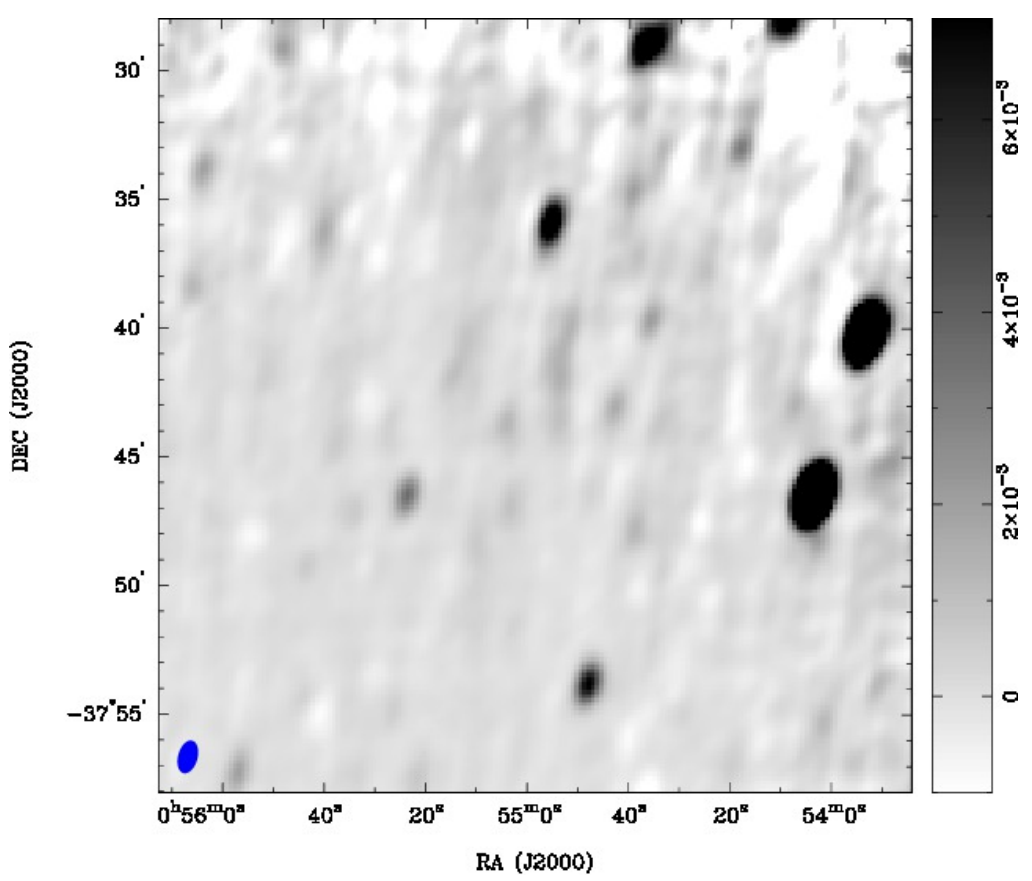

Fig. 6 VLA Project AC0308 radio-continuum total intensity image of NGC 300. The synthesised beam, as represented by the blue ellipse in the lower left hand corner, is $77.8^{\prime \prime} \times 44.7^{\prime \prime}$ and the r.m.s. noise is $0.50 \mathrm{mJy} /$ beam. This image is in terms of Jy/Beam.

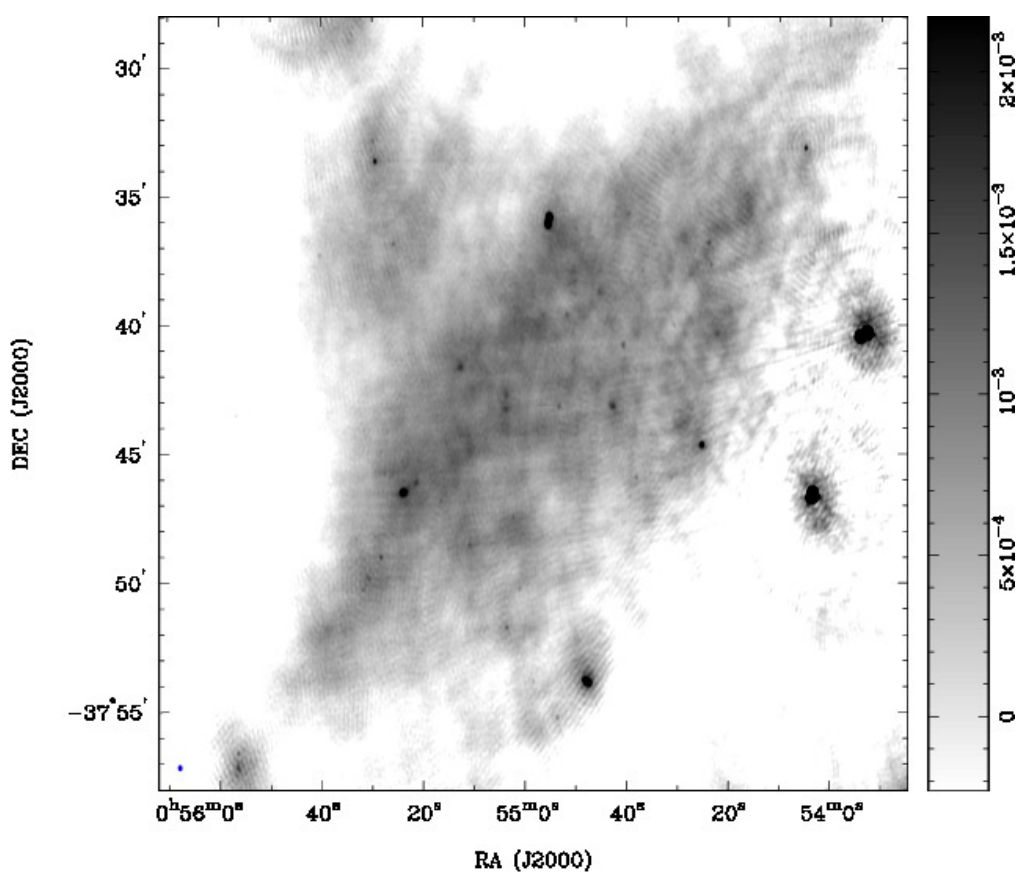

Fig. 7 Combined ATCA projects C1757 and C828 mosaic radio-continuum total intensity image of NGC 300. The synthesised beam, as represented by the blue ellipse in the lower left hand corner, is $13.8^{\prime \prime} \times 10.1^{\prime \prime}$ and the r.m.s noise is $0.07 \mathrm{mJy} /$ beam. This image is in terms of Jy/Beam. 


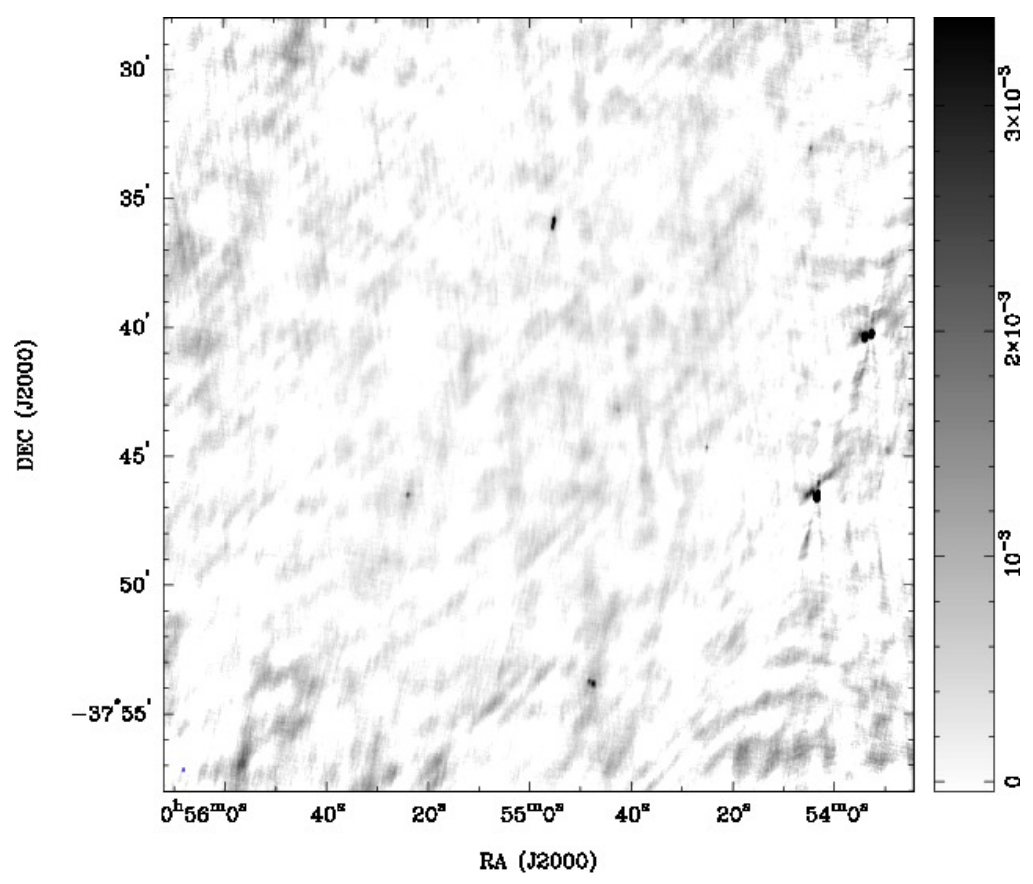

Fig. 8 Combined VLA projects AL445, AC101 and AC308 mosaic radio-continuum total intensity image of NGC 300. The synthesised beam, as represented by the blue ellipse in the lower left hand corner, is $10.9^{\prime \prime} \times 4.8^{\prime \prime}$ and the r.m.s. noise is $0.09 \mathrm{mJy} /$ beam. This image is in terms of $\mathrm{Jy} /$ Beam.

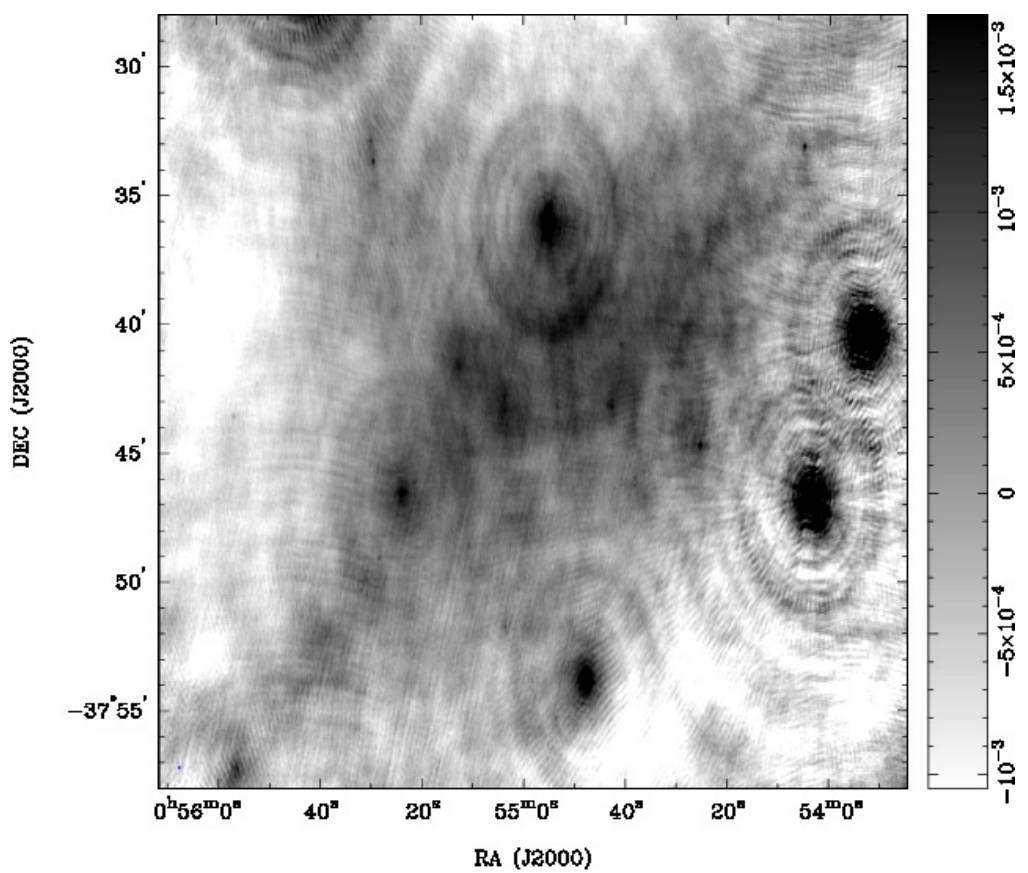

Fig. 9 All ATCA and VLA projects radio-continuum total intensity image of NGC 300. The synthesised beam, as represented by the blue ellipse in the lower left hand corner, is $11.8^{\prime \prime} \times 5.0^{\prime \prime}$ and the r.m.s. noise is $0.13 \mathrm{mJy} / \mathrm{beam}$. This image is in terms of Jy/Beam. 


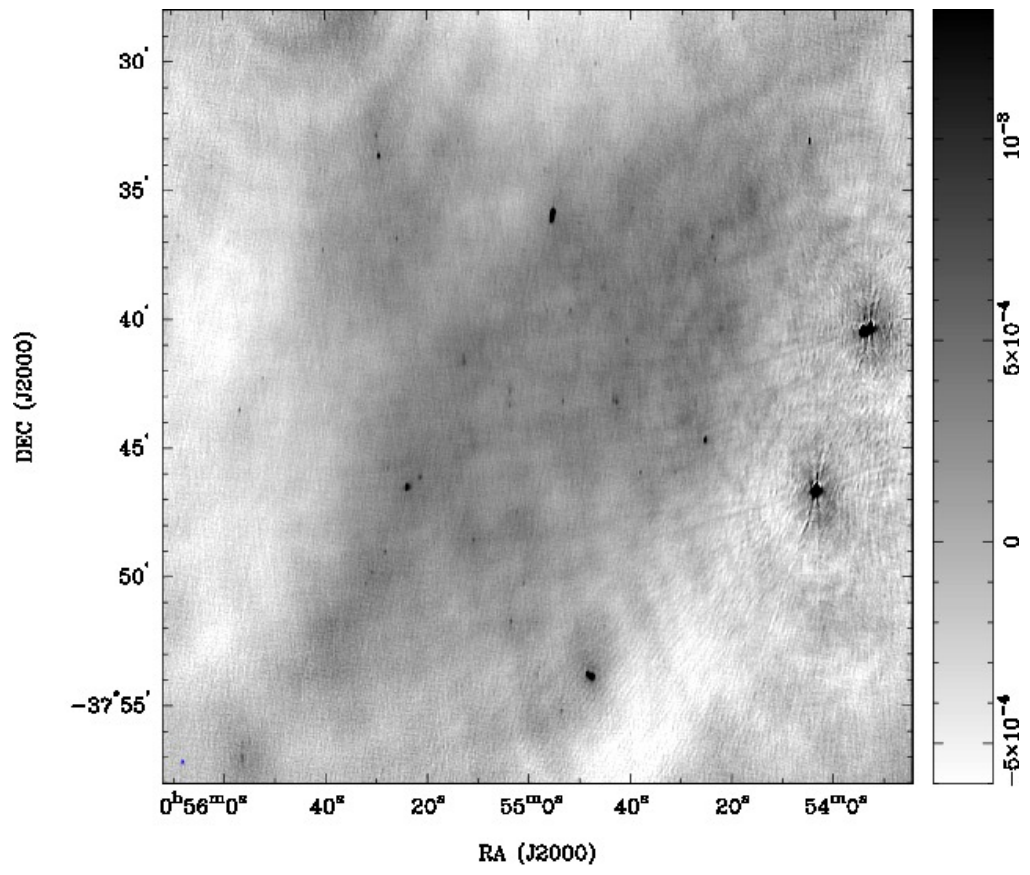

Fig. 10 All ATCA and VLA projects radio-continuum total intensity image of NGC 300 produced using the MIRIAD task IMMERGE. The synthesised beam, as represented by the blue ellipse in the lower left hand corner, is $10.8^{\prime \prime} \times 4.8^{\prime \prime}$ and the r.m.s. noise is $0.06 \mathrm{mJy} /$ beam. This image is in terms of Jy/Beam.

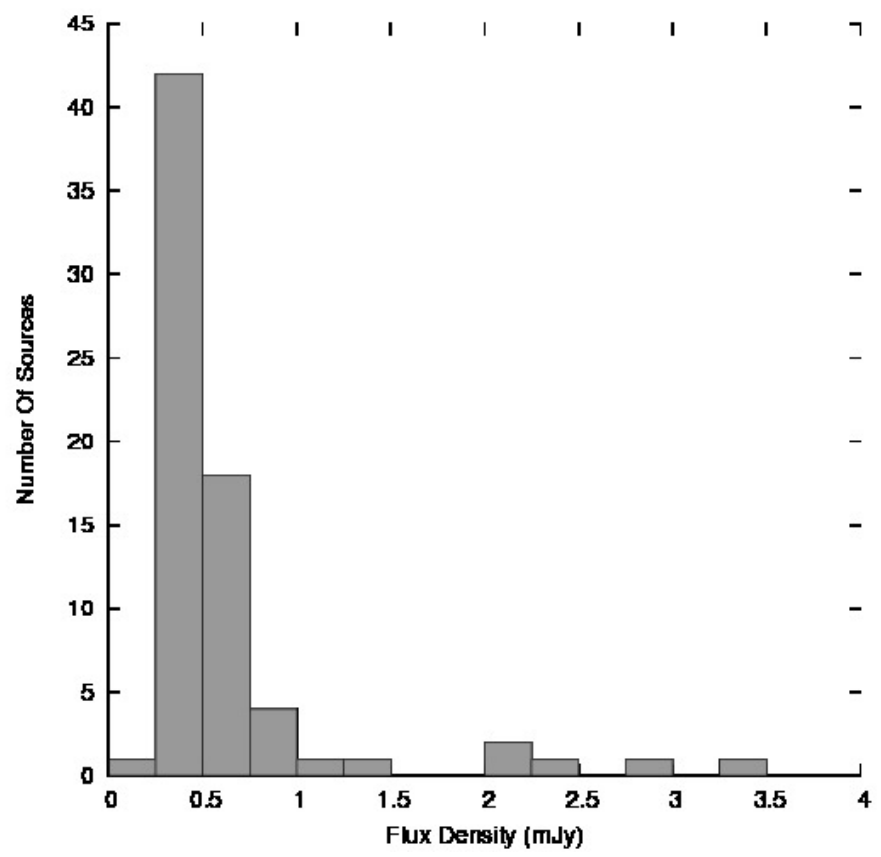

Fig. 11 A histogram of source flux density distribution within the field of NGC 300 . Source No. 42 from Table 3 is excluded from this graph. 
in common with the ATCA observations (Col. 5), our flux densities are higher by $18 \pm 6 \%$; for the 33 sources in common with VLA observations (Col. 6), our flux densities are higher by $19 \pm 7 \%$.

The discrepancy between the flux density measurements is likely to be due to the extended continuum emission discussed below. In the earlier study, this component was resolved out of the images, and therefore not measured in the point-source flux densities, whereas our image includes this emission, and our point-source flux densities also include it.

\subsection{Extended Radio-continuum Emission from NGC 300}

Fig. 12 shows a DSS2 optical image of NGC 300 overlaid with our final radio-continuum contours from Fig. 10 The structure of the radio emission from NGC 300 is significantly different from that of its optical emission.

Although the radio overlay aligns with a number of sources found in the optical image, there is a clear region of radio-continuum emission continuing past the apparent optical boundaries of NGC 300 and at a different position angle. The radio-continuum contours are consistent with the existence and positioning of the $\mathrm{HI}$ outer disc spanning more than $1^{\circ}$ (equivalent to about $35 \mathrm{kpc}$ ) across the sky (Westmeier et al. 2011). They suggested that there is a substantial change in the position angle between the inner and the outer disc, resulting in a twisted appearance of NGC 300 which is particularly obvious in the velocity field. One possible scenario for this twist is that the distortion and warping of the outer disc of NGC 300 was caused by tidal forces during a recent encounter with another galaxy, with nearby NGC 45 as a potential candidate for a close encounter.

While it is very difficult to precisely estimate the total flux density of NGC 300 extended radio-continuum emission at $20 \mathrm{~cm}$ we selected a tight region around $3 \sigma$ contour seen in Fig. 12 and we summed all intensity inside this region to an integrated flux density of $4.687 \pm 0.005 \mathrm{Jy}$. This includes the extended emission to the north-east of the main disk. The total flux density of all point sources detected within this region is $0.067 \pm 0.005 \mathrm{Jy}$, so we estimate the flux density of the extended emission to be $4.62 \pm 0.01 \mathrm{Jy}$.

\subsection{Source Classification}

Thirty-five radio-continuum sources previously classified by Payne et al. (2004) were detected in our study. These sources are classified into three groups: background sources (such as AGN), Supernova Remnants
(SNRs) and H II regions. To determine the true nature of the newly discovered sources from this survey, we used the previous classification scheme in addition to comparison with the available optical images such as DSS2.

We found 8 new sources (marked as lower case $y$ in Table 3. Col. 7) that are in common with optical and radio images making a total of 24 sources in common between optical and radio surveys. These 8 newly detected optical sources are most likely to originate in NGC 300 as either SNRs or H II regions. Because of their prominent optical extended emission, two sources (Table 3 No. 40 and 53) which were previously classified as background AGN's are more likely to be intrinsic to NGC 300. We also compared our new radio catalogue with existing NGC 300 IR Spitzer images (Helou et al. 2004) at all frequencies $(3.6,4.5,5.8,8.024,70$ and $150 \mu \mathrm{m})$. We found 37 sources in common between radio and various IR frequencies. In total, 21 sources are common to all three surveys. Combining previous work and the new radio maps we classify 51 objects. From these 51 sources some 29 are most likely to be $\mathrm{H}$ II regions, four are SNRs and one is an X-ray binary. We classify eleven sources as background sources (Table 3 No. 9, 17, 37, 42, 44, 47, 49, 60, 62, 63 and 64 where 42 is a double source) and six sources being within NGC 300 (SNRs or H II). Finally, the remaining 21 radio-continuum sources are still not classified.

We estimated the flux density of a bright Galactic SNR (such as Cas A) at a distance of $1.9 \mathrm{Mpc}$, and compared this to our radio point source catalogue; we find that there are no Cas A analogues in NGC 300. The luminous radio SNR source \# 62 (at about $2 \mathrm{mJy}$ ) is twice that of a Crab Nebula analogue (which would be about a $1 \mathrm{mJy}$ source in NGC 300). These results are similar to those in Pannuti et al. (2000).

\section{CONCLUSION}

We present and discuss our new high-sensitivity and high-resolution radio-continuum images of NGC 300 at $20 \mathrm{~cm}$. The new images were created from merging sensitive 20-cm mosaic radio surveys from ATCA, and from the VLA synthesis radio-telescope. We were able to dramatically improve both the sensitivity and resolution of the final radio-continuum image when compared to previous studies. This resulted in the identification of a number of previously unknown sources bringing the number of known radio sources within the area of NGC 300 to 72 . We also detected a previously unseen region of NGC 300's extended radio-continuum emission estimated to have an integrated flux density 
Table 3 List of sources at $20 \mathrm{~cm}$ within the field of NGC 300. Columns 1, 2, 3 and 4 are from this work. Columns 5 and 6 are partially extracted from Payne et al. (2004). Columns 7 and 8 correspond to the DSS2 (optical) and Spitzer (IR) identification.

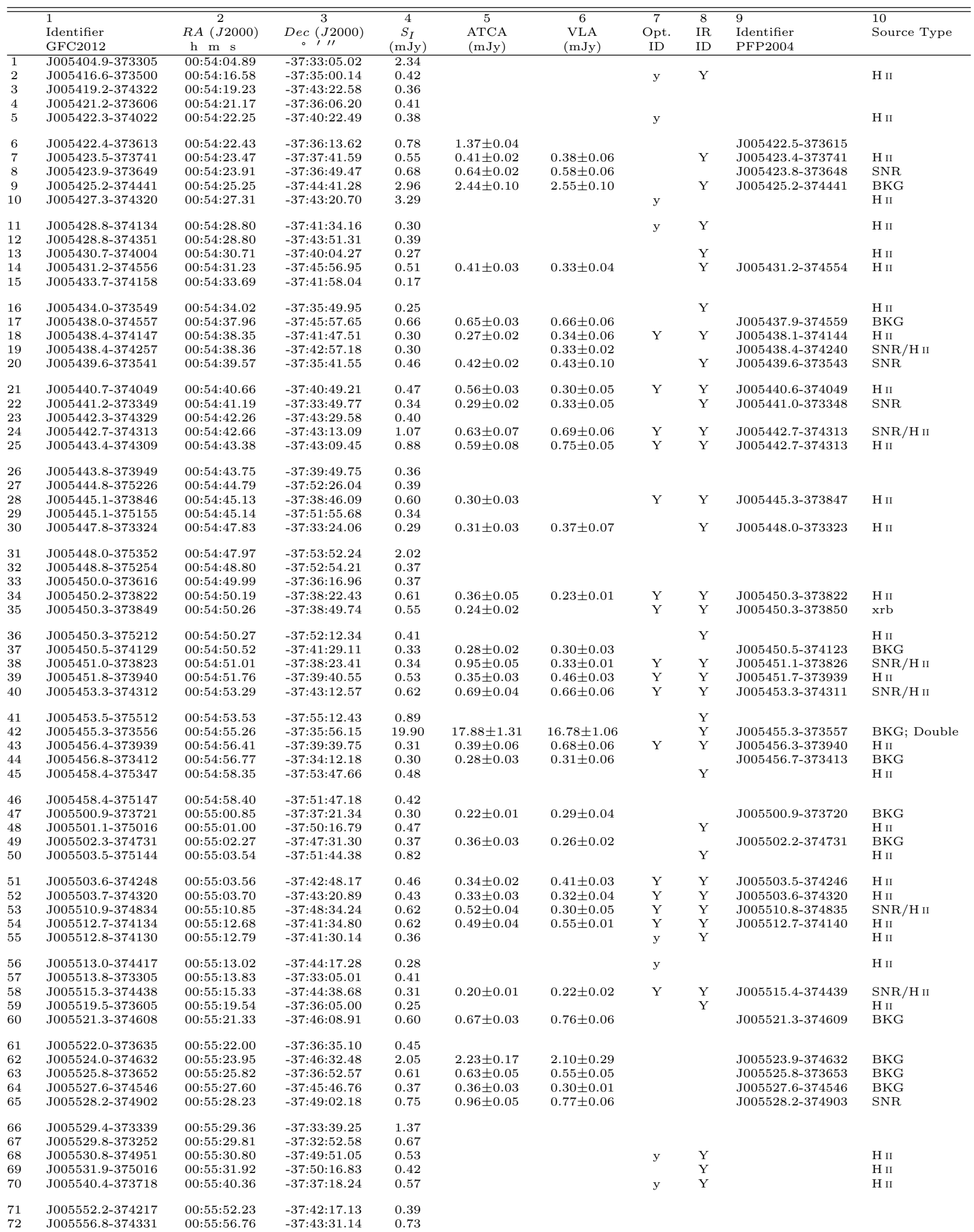




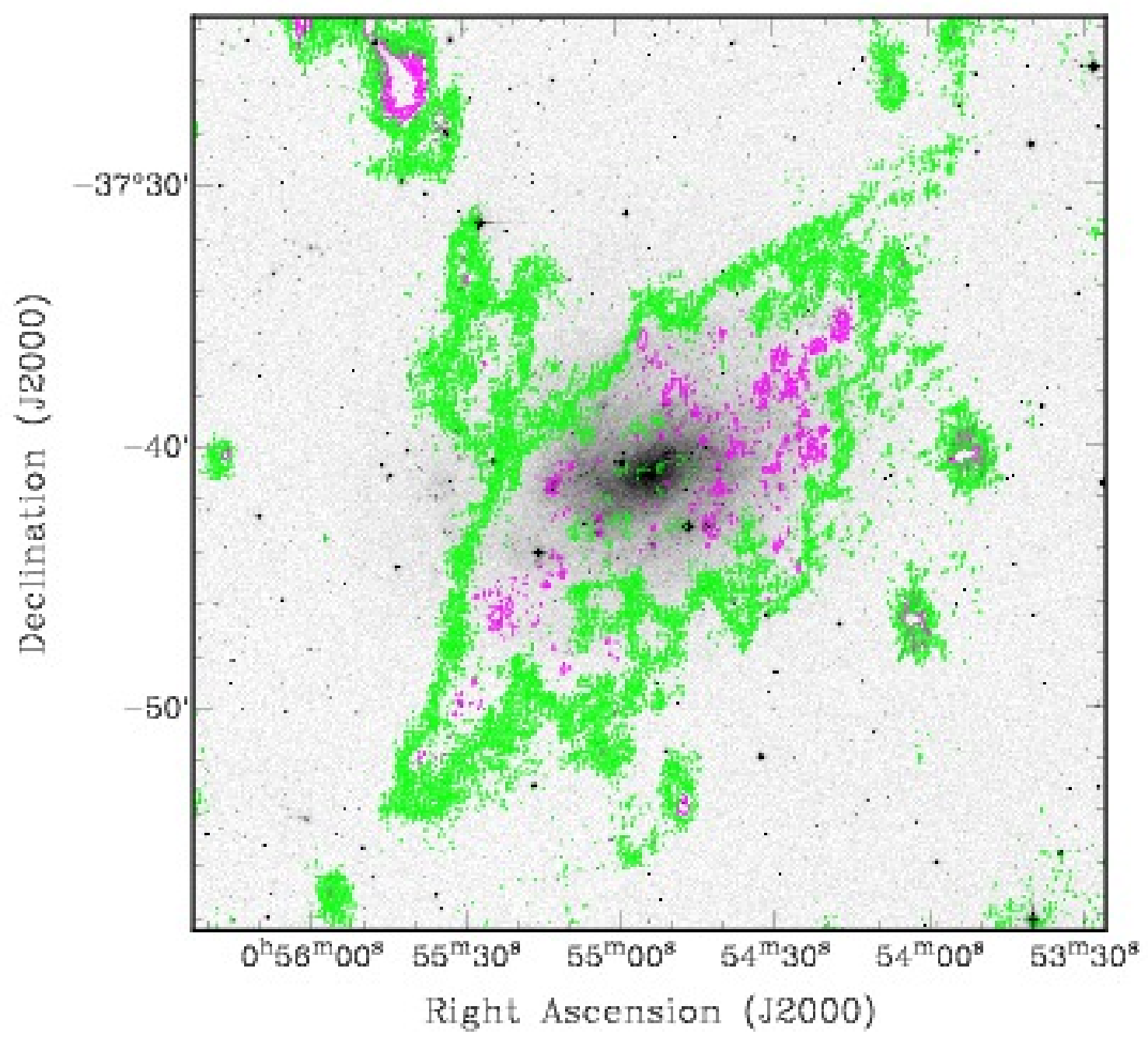

Fig. 12 An optical image of NGC 300 overlaid with our final radio-continuum image from Fig. 10. The overlaid contours are $0.21(3 \sigma$; green) and $0.49 \mathrm{mJy} /$ beam (magenta). 
of $4.62 \pm 0.01 \mathrm{Jy}$. It was also demonstrated that a joint deconvolved image produced by both ATCA and VLA observations Fig. 9 was inferior to an image produced with an IMmerge (Fig. 10) approach. We believe that this difference is attributed to MIRIAD's inability to produce an accurate beam representation when datasets from multiple telescopes are jointly inverted. Existing sidelobes are exaggerated when cleaning the resulting dirty map because of the inaccurate beam.

\section{Acknowledgments}

The National Radio Astronomy Observatory is a facility of the National Science Foundation operated under cooperative agreement by Associated Universities, Inc. The Australia Telescope Compact Array is part of the Australia Telescope National Facility which is funded by the Commonwealth of Australia for operation as a National Facility managed by CSIRO. This paper includes archived data obtained through the Australia Telescope Online Archive (http://atoa.atnf.csiro.au) and VLA.

We would also like to thank the anonymous referee, whose feedback greatly improved this paper.

\section{References}

Bresolin, F., Gieren, W., Kudritzki, R.-P., Pietrzyński, G., Urbaneja, M.A., Carraro, G.: Astrophys. J. 700, 309 (2009). doi:10.1088/0004-637X/700/1/309

Gooch, R.: Karma Users Guide. ATNF, Sydney (2006)

Greisen, E. (ed.): Aips Cookbook. The National Radio Astronomy Observatory, Charlottesville (2010)

Helou, G., Roussel, H., Appleton, P., Frayer, D., Stolovy, S., Storrie-Lombardi, L., Hurt, R., Lowrance, P., Makovoz, D., Masci, F., Surace, J., Gordon, K.D., Alonso-Herrero, A., Engelbracht, C.W., Misselt, K., Rieke, G., Rieke, M., Willner, S.P., Pahre, M., Ashby, M.L.N., Fazio, G.G., Smith, H.A.: Astrophys. J. Suppl. Ser. 154, 253 (2004). doi: $10.1086 / 422640$

Millar, W.C., White, G.L., Filipović, M.D., Payne, J.L., Crawford, E.J., Pannuti, T.G., Staggs, W.D.: Astrophys. Space Sci. 332, 221 (2011). doi:10.1007/s10509-010-0556y

Pannuti, T.G., Duric, N., Lacey, C.K., Goss, W.M., Hoopes, C.G., Walterbos, R.A.M., Magnor, M.A.: Astrophys. J. 544, 780 (2000). doi:10.1086/317238

Payne, J.L., Filipović, M.D., Pannuti, T.G., Jones, P.A., Duric, N., White, G.L., Carpano, S.: Astron. Astrophys. 425, 443 (2004). doi:10.1051/0004-6361:20041058

Rizzi, L., Bresolin, F., Kudritzki, R., Gieren, W., Pietrzyński G.: Astrophys. J. 638, 766 (2006). doi:10.1086/498705

Sault, R., Killeen, N.: Miriad Users Guide. ATNF, Sydney (2006)
Steer, D.G., Dewdney, P.E., Ito, M.R.: Astron. Astrophys. 137, 159 (1984)

Westmeier, T., Braun, R., Koribalski, B.S.: Mon. Not. R. Astron. Soc. 410, 2217 (2011). doi:10.1111/j.13652966.2010.17596.x 